\title{
RANK PROPERTY OF THE MIMO GAUSSIAN WIRETAP CHANNEL WITH AN AVERAGE POWER CONSTRAINT
}

\author{
S. Ali. A. Fakoorian, Jing Huang, A. Lee Swindlehurst \\ Center for Pervasive Communications and Computing \\ University of California Irvine \\ afakoori, jing.huang, swindle@uci.edu
}

\begin{abstract}
This paper considers a multiple-input multiple-output (MIMO) Gaussian wiretap channel, where there exists a transmitter, a legitimate receiver and an eavesdropper, each equipped with multiple antennas. In particular, we study the rank of the optimal input covariance matrix that achieves the secrecy capacity of the multiple antenna MIMO Gaussian wiretap channel under an average power constraint. The rank and other properties of the optimal solution are derived based on certain relationships between the channel matrices for the legitimate receiver and eavesdropper. Such properties are useful steps towards characterizing the general solution to the MIMO wiretap problem with an average power constraint.
\end{abstract}

Index Terms - MIMO Wiretap Channel, Secrecy Capacity, Physical Layer Security

\section{INTRODUCTION}

The broadcast nature of a wireless medium makes it very susceptible to eavesdropping, where the transmitted message is decoded by unintended receiver(s). Recent informationtheoretic research on secure communication has focused on enhancing security at the physical layer. The wiretap channel, first introduced and studied by Wyner [1], is the most basic physical layer model that captures the problem of communication security. Wyner showed that when an eavesdropper's channel is a degraded version of the main channel, the source and destination can achieve a positive secrecy rate, while ensuring that the eavesdropper receives zero bits of information. The maximum secrecy rate from the source to the destination is defined as the secrecy capacity. The Gaussian wiretap channel, in which the outputs at the legitimate receiver and at the eavesdropper are corrupted by additive white Gaussian noise, was studied in [2].

Determining the secrecy capacity of a Gaussian MIMO wiretap channel is in general a difficult non-convex optimization problem, and has been addressed independently in [3]-

This work was supported by the U.S. Army Research Office under the Multi-University Research Initiative (MURI) grant W911NF-07-1-0318, and by the National Science Foundation under grant CCF-1117983.
[9]. Oggier and Hassibi [3] and Khisti and Wornell [4] followed an indirect approach using a Sato-like argument and matrix analysis tools. They considered the problem of finding the secrecy capacity under a constraint on the average total power, and a closed-form expression for the secrecy capacity in the high signal-to-noise-ratio (SNR) regime was obtained in [4]. The rank of the optimal input covariance matrix for the secrecy rate maximization problem is discussed in [5], but the authors were unable to characterize the solution for the general case. For some special cases of the MIMO wiretap channel, where the solution has rank one, the optimal input covariance matrix that achieves the secrecy capacity under the average total power constraint was obtained in [5]-[7].

In [8], Liu and Shamai propose a more informationtheoretic approach using the enhancement concept, originally presented by Weingarten et al. [10], as a tool for the characterization of the MIMO Gaussian broadcast channel capacity. Liu and Shamai have shown that an enhanced degraded version of the channel attains the same secrecy capacity as does a Gaussian input distribution. From the mathematical solution in [8] it was evident that such an enhanced channel exists; however it was not clear how to construct such a channel until the work of [9], which provided a closed-form expression for the secrecy capacity under an input covariance matrix constraint. While this result is interesting since the expression for the secrecy capacity is valid for all SNR scenarios, there still exists no computable secrecy capacity expression for the MIMO Gaussian wiretap channel under an average total power constraint.

In this paper, we investigate the rank of the optimal input covariance matrix that achieves the secrecy capacity of the general Gaussian multiple-input multiple-output (MIMO) wiretap channel under the average total power constraint, where the number of antennas is arbitrary for both the transmitter and the two receivers. Throughout this analysis, other interesting properties for the optimal input covariance matrix are revealed as well.

Notation: Throughout the paper, boldface uppercase letters are used to denote matrices, and vector-valued random variables are written with non-boldface uppercase letters (e.g., 
$X$ ), while the corresponding non-boldface lowercase letter (x) denotes a specific realization of the random variable. Scalar variables are written with non-boldface (lowercase or uppercase) letters. The Hermtian (i.e., conjugate) transpose is denoted by $(.)^{H}$, the matrix trace by $\operatorname{Tr}($.$) , and \mathbf{I}$ indicates an identity matrix. Inequality $\mathbf{A} \preceq \mathbf{B}$ means that $\mathbf{A}-\mathbf{B}$ is Hermitian positive semi-definite. Mutual information between the random variables $A$ and $B$ is denoted by $I(A ; B)$, $E$ is the expectation operator, and $\mathcal{C N}\left(0, \sigma^{2}\right)$ represents the complex circularly symmetric Gaussian distribution with zero mean and variance $\sigma^{2}$. The projection matrix onto the column space of $\mathbf{X}$ is denoted by $\mathbf{P}_{\mathbf{X}}=\mathbf{X}\left(\mathbf{X}^{H} \mathbf{X}\right)^{-1} \mathbf{X}^{H}$, and $\mathbf{P}_{\mathbf{X}}^{\perp}=\mathbf{I}-\mathbf{P}_{\mathbf{X}}$ denotes the projection onto the space orthogonal to $\mathbf{X} \cdot \operatorname{span}\{\mathbf{X}\}$ denotes the space spanned by the column vectors of $\mathbf{X}$, and $\operatorname{span}\{\mathbf{X}\}^{\perp}$ denotes the orthogonal complement space of $\operatorname{span}\{\mathbf{X}\}$.

\section{SYSTEM MODEL AND PRIOR WORK}

We begin with a multiple-antenna wiretap channel with $n_{t}$ transmit antennas and $n_{r}$ and $n_{e}$ receive antennas at the legitimate recipient and the eavesdropper, respectively:

$$
\begin{aligned}
& \mathbf{y}_{r}=\mathbf{H x}+\mathbf{z}_{r} \\
& \mathbf{y}_{e}=\mathbf{G x}+\mathbf{z}_{e}
\end{aligned}
$$

where $\mathbf{x}$ is a zero-mean $n_{t} \times 1$ transmitted signal vector, $\mathbf{z}_{r} \in$ $\mathbb{C}^{n_{r} \times 1}$ and $\mathbf{z}_{e} \in \mathbb{C}^{n_{e} \times 1}$ are additive white Gaussian noise vectors at the receiver and eavesdropper, respectively, with i.i.d. entries distributed as $\mathcal{C N}(0,1)$. The matrices $\mathbf{H} \in$ $\mathbb{C}^{n_{r} \times n_{t}}$ and $\mathbf{G} \in \mathbb{C}^{n_{e} \times n_{t}}$ represent the channels associated with the receiver and the eavesdropper, respectively. Similar to other papers considering the perfect secrecy rate of the wiretap channel, we assume that the transmitter has perfect channel state information (CSI) for both the legitimate receiver and the eavesdropper. For the Gaussian channel, where Gaussian inputs are an optimal choice, the secrecy capacity is given by [3]

$$
\mathcal{C}_{\text {sec }}=\max _{\mathbf{x}}\left[I\left(X ; Y_{r}\right)-I\left(X ; Y_{e}\right)\right]=\max _{\mathbf{Q} \succeq \mathbf{0}} R(\mathbf{Q})
$$

where $R(\mathbf{Q})=\log \left|\mathbf{H Q H} \mathbf{H}^{H}+\mathbf{I}\right|-\log \left|\mathbf{G Q G} \mathbf{G}^{H}+\mathbf{I}\right|$, and $\mathbf{Q}=E\left\{\mathbf{x x}^{H}\right\}$ is the input covariance matrix. The maximization problem in (2) is on the channel input, where the channel input is under either an average power constraint or an input covariance matrix constraint, as described below.

In [9], the above secret communication problem was analyzed under the input covariance constraint, defined as

$$
\begin{aligned}
\mathcal{C}_{\text {sec }}(\mathbf{S})= & \max \log \left|\mathbf{H Q H} \mathbf{H}^{H}+\mathbf{I}\right|-\log \left|\mathbf{G Q G} \mathbf{G}^{H}+\mathbf{I}\right| \\
& \text { s.t. } \mathbf{Q} \preceq \mathbf{S}
\end{aligned}
$$

where $\mathbf{S}$ is a positive semi-definite matrix that defines the input covariance constraint. An explicit expression for the secrecy capacity under (3) was obtained via applying the generalized eigenvalue decomposition to the following two positive definite matrices

$$
\left(\mathbf{S}^{\frac{1}{2}} \mathbf{H}^{H} \mathbf{H} \mathbf{S}^{\frac{1}{2}}+\mathbf{I} \quad, \quad \mathbf{S}^{\frac{1}{2}} \mathbf{G}^{H} \mathbf{G} \mathbf{S}^{\frac{1}{2}}+\mathbf{I}\right) .
$$

In particular, there exists an invertible generalized eigenvector matrix $\mathbf{C}$ such that [12]

$$
\begin{array}{r}
\mathbf{C}^{H}\left[\mathbf{S}^{\frac{1}{2}} \mathbf{G}^{H} \mathbf{G S}^{\frac{1}{2}}+\mathbf{I}\right] \mathbf{C}=\mathbf{I} \\
\mathbf{C}^{H}\left[\mathbf{S}^{\frac{1}{2}} \mathbf{H}^{H} \mathbf{H} \mathbf{S}^{\frac{1}{2}}+\mathbf{I}\right] \mathbf{C}=\boldsymbol{\Lambda}
\end{array}
$$

where $\boldsymbol{\Lambda}=\operatorname{diag}\left\{\lambda_{1}, \ldots, \lambda_{n_{t}}\right\}$ is a positive definite diagonal matrix and $\lambda_{1}, \ldots, \lambda_{n_{t}}$ represent the generalized eigenvalues. Without loss of generality, we assume the eigenvalues are ordered as

$$
\lambda_{1} \geq \ldots \geq \lambda_{b}>1 \geq \lambda_{b+1} \geq \ldots \geq \lambda_{n_{t}}>0
$$

so that a total of $b\left(0 \leq b \leq n_{t}\right)$ are greater than 1 . Hence, we can write $\boldsymbol{\Lambda}$ as

$$
\boldsymbol{\Lambda}=\left[\begin{array}{cc}
\boldsymbol{\Lambda}_{1} & \mathbf{0} \\
\mathbf{0} & \boldsymbol{\Lambda}_{2}
\end{array}\right]
$$

where $\boldsymbol{\Lambda}_{1}=\operatorname{diag}\left\{\lambda_{1}, \ldots, \lambda_{b}\right\}$ and $\boldsymbol{\Lambda}_{2}=\operatorname{diag}\left\{\lambda_{b+1}, \ldots, \lambda_{n_{t}}\right\}$. We can partition $\mathbf{C}$ similarly:

$$
\mathbf{C}=\left[\begin{array}{ll}
\mathbf{C}_{1} & \mathbf{C}_{2}
\end{array}\right]
$$

where $\mathbf{C}_{1}$ is the $n_{t} \times b$ submatrix representing the generalized eigenvectors corresponding to $\left\{\lambda_{1}, \ldots, \lambda_{b}\right\}$ and $\mathbf{C}_{2}$ is the $n_{t} \times$ $\left(n_{t}-b\right)$ submatrix representing the generalized eigenvectors corresponding to $\left\{\lambda_{b+1}, \ldots, \lambda_{n_{t}}\right\}$.

Using the above notation, the secrecy capacity of the MIMO wiretap channel under the input covariance constraint (3) can be expressed as [9], [11, Theorem 3]:

$$
\mathcal{C}_{\text {sec }}(\mathbf{S})=\sum_{i=1}^{b} \log \lambda_{i}=\log \left|\boldsymbol{\Lambda}_{1}\right|
$$

where the optimal input covariance matrix $\mathbf{Q}_{S}^{*}$ that maximizes (2) and attains (9) is given by

$$
\mathbf{Q}_{S}^{*}=\mathbf{S}^{\frac{1}{2}} \mathbf{C}\left[\begin{array}{cc}
\left(\mathbf{C}_{1}^{H} \mathbf{C}_{1}\right)^{-1} & \mathbf{0} \\
\mathbf{0} & \mathbf{0}
\end{array}\right] \mathbf{C}^{H} \mathbf{S}^{\frac{1}{2}}
$$

Remark 1 From (5) and (6), one can easily confirm that if $\mathbf{H}^{H} \mathbf{H} \preceq \mathbf{G}^{H} \mathbf{G}$, then for any $\mathbf{S} \succeq \mathbf{0}$ we have $\mathbf{\Lambda} \preceq \mathbf{I}$. In other words, in this case the pencil in (4) has no generalized eigenvalue bigger than 1. Thus, $\mathcal{C}_{\text {sec }}(\mathbf{S})=0$ for any $\mathbf{S} \succeq \mathbf{0}$.

Lemma 1 For the case of $\mathbf{H}^{H} \mathbf{H} \succ \mathbf{G}^{H} \mathbf{G}$, for any $n_{t} \times n_{t}$ matrix $\mathbf{S} \succeq \mathbf{0}$, all the generalized eigenvalues of the pencil $\left(\mathbf{S}^{\frac{1}{2}} \mathbf{H}^{H} \mathbf{H S}^{\frac{1}{2}}+\mathbf{I}, \mathbf{S}^{\frac{1}{2}} \mathbf{G}^{H} \mathbf{G S}^{\frac{1}{2}}+\mathbf{I}\right)$ are strictly bigger than 1, i.e. $\boldsymbol{\Lambda} \succ \mathbf{I}$, iff $\mathbf{S}$ is full rank, i.e. $\mathbf{S} \succ \mathbf{0}$. 
Proof: Subtracting (5) from (6), a straightforward computation yields

$$
\mathbf{S}^{\frac{1}{2}}\left(\mathbf{H}^{H} \mathbf{H}-\mathbf{G}^{H} \mathbf{G}\right) \mathbf{S}^{\frac{1}{2}}=\mathbf{C}^{-H}(\boldsymbol{\Lambda}-\mathbf{I}) \mathbf{C}^{-1} .
$$

When $\mathbf{H}^{H} \mathbf{H} \succ \mathbf{G}^{H} \mathbf{G}$, both sides of the above equality are positive semi-definite; i.e., for any $\mathbf{S} \succeq \mathbf{0}$ we must have $\boldsymbol{\Lambda} \succeq$ I. If $\mathbf{S} \succ \mathbf{0}$, the left side is full-rank and thus $\boldsymbol{\Lambda} \succ \mathbf{I}$, and vice versa.

In this paper, we consider the secrecy capacity problem in (2) under the average power constraint:

$$
\begin{gathered}
\mathcal{C}_{\text {sec }}\left(P_{t}\right)=\max \log \left|\mathbf{H Q H} \mathbf{H}^{H}+\mathbf{I}\right|-\log \left|\mathbf{G} \mathbf{Q} \mathbf{G}^{H}+\mathbf{I}\right| \\
\text { s.t. } \operatorname{Tr}\left(E\left\{\mathbf{x} \mathbf{x}^{H}\right\}\right)=\operatorname{Tr}(\mathbf{Q}) \leq P_{t} .
\end{gathered}
$$

For this constraint, no computable secrecy capacity expression has been derived to date for the general MIMO case. It was shown in [10, Lemma 1], [11] that, the wiretap channel under the average power constraint (11) is equivalent to the channel under the input covariance constraint (3) through an exhaustive search over the set $\{\mathbf{S}: \mathbf{S} \succeq \mathbf{0}, \operatorname{Tr}(\mathbf{S}) \leq P\}$ :

$$
\mathcal{C}_{s e c}\left(P_{t}\right)=\max _{\mathbf{S} \succeq \mathbf{0}, \operatorname{Tr}(\mathbf{S}) \leq P_{t}} \mathcal{C}_{s e c}(\mathbf{S})
$$

where for any given semidefinite $\mathbf{S}, \mathcal{C}_{\text {sec }}(\mathbf{S})$ should be computed as given by (9). Also note that for any covariance constraint $\mathbf{S}$, the optimal input covariance matrix $\mathbf{Q}_{S}^{*}$ that attains $\mathcal{C}_{\text {sec }}(\mathbf{S})$ is given by (10), and $\mathbf{Q}_{S}^{*} \preceq \mathbf{S}$.

In the next section, we investigate the rank of the optimal input covariance matrix $\mathbf{Q}^{*}$ that attains $\mathcal{C}_{s e c}\left(P_{t}\right)$.

\section{PROPERTIES OF THE OPTIMAL SOLUTION UNDER AN AVERAGE POWER CONSTRAINT}

For the following results in the paper, we exclude the special case $\mathbf{H}^{H} \mathbf{H} \preceq \mathbf{G}^{H} \mathbf{G}$ for which the $C_{\text {sec }}$ is trivially 0 for any $\mathbf{S} \succeq \mathbf{0}$, and consequently for any $P_{t}$, as pointed out in Remark 1. Also we note that problem (11) under $\operatorname{Tr}(\mathbf{Q}) \leq P_{t}$ is equivalent to that under $\operatorname{Tr}(\mathbf{Q})=P_{t}$, see [3, Eq. (9)], [5]. Considering (11) in the form of (12), and recalling that for any $\mathbf{S}, \mathbf{Q}_{S}^{*} \preceq \mathbf{S}$, thus $\operatorname{Tr}\left(\mathbf{Q}_{S}^{*}\right) \leq \operatorname{Tr}(\mathbf{S})$, the above point implies that we can restrict the set of input covariance constraints to $\mathbf{S} \succeq \mathbf{0}, \operatorname{Tr}(\mathbf{S})=P_{t}$ instead of $\operatorname{Tr}(\mathbf{S}) \leq P_{t}$.

\section{1. span properties}

Let $\widehat{\mathbf{S}}$ denote the input covariance constraint that maximizes (12). Consequently, from (10), the optimal input covariance matrix that attains $\mathcal{C}_{s e c}\left(P_{t}\right)$ is given by ${ }^{1}$

$$
\mathbf{Q}^{*}=\widehat{\mathbf{S}}^{\frac{1}{2}} \widehat{\mathbf{C}}\left[\begin{array}{cc}
\left(\widehat{\mathbf{C}}_{1}^{H} \widehat{\mathbf{C}}_{1}\right)^{-1} & \mathbf{0} \\
\mathbf{0} & \mathbf{0}
\end{array}\right] \widehat{\mathbf{C}}^{H} \widehat{\mathbf{S}}^{\frac{1}{2}}
$$

\footnotetext{
${ }^{1}$ To be consistent with notation in (10), the optimal input covariance matrix under the covariance constraint $\widehat{\mathbf{S}}$ should be written as $\mathbf{Q}_{\widehat{S}}^{*}$, which for simplicity is denoted just as $\mathbf{Q}^{*}$.
}

where $\widehat{\mathbf{C}}$ and $\widehat{\mathbf{C}}_{1}$ have respectively the same definitions as those of $\mathbf{C}$ and $\mathbf{C}_{1}$, given by (5)-(8), but here for the pencil $\left(\widehat{\mathbf{S}}^{\frac{1}{2}} \mathbf{H}^{H} \mathbf{H} \widehat{\mathbf{S}}^{\frac{1}{2}}+\mathbf{I}, \widehat{\mathbf{S}}^{\frac{1}{2}} \mathbf{G}^{H} \mathbf{G} \widehat{\mathbf{S}}^{\frac{1}{2}}+\mathbf{I}\right)$. Note that $\mathbf{Q}^{*}$ can be rewritten as

$$
\begin{aligned}
\mathbf{Q}^{*} & =\widehat{\mathbf{S}}^{\frac{1}{2}}\left[\begin{array}{ll}
\widehat{\mathbf{C}}_{1} & \widehat{\mathbf{C}}_{2}
\end{array}\right]\left[\begin{array}{cc}
\left(\widehat{\mathbf{C}}_{1}^{H} \widehat{\mathbf{C}}_{1}\right)^{-1} & \mathbf{0} \\
\mathbf{0} & \mathbf{0}
\end{array}\right]\left[\begin{array}{l}
\widehat{\mathbf{C}}_{1}^{H} \\
\widehat{\mathbf{C}}_{2}^{H}
\end{array}\right] \widehat{\mathbf{S}}^{\frac{1}{2}} \\
& =\widehat{\mathbf{S}}^{\frac{1}{2}} \widehat{\mathbf{C}}_{1}\left(\widehat{\mathbf{C}}_{1}^{H} \widehat{\mathbf{C}}_{1}\right)^{-1} \widehat{\mathbf{C}}_{1}^{H} \widehat{\mathbf{S}}^{\frac{1}{2}} \\
& =\widehat{\mathbf{S}}^{\frac{1}{2}} \mathbf{P}_{\widehat{\mathbf{C}}_{1}} \widehat{\mathbf{S}}^{\frac{1}{2}}
\end{aligned}
$$

where $\mathbf{P}_{\widehat{\mathbf{C}}_{1}}=\widehat{\mathbf{C}}_{1}\left(\widehat{\mathbf{C}}_{1}^{H} \widehat{\mathbf{C}}_{1}\right)^{-1} \widehat{\mathbf{C}}_{1}^{H}$ is the projection matrix onto the space of $\widehat{\mathbf{C}}_{1}$. Moreover, let $\mathbf{P}{\widehat{\mathbf{C}}_{1}}_{\widehat{\mathbf{C}}_{1}}^{\perp}=\mathbf{I}-\mathbf{P}_{\widehat{\mathbf{C}}_{1}}$ be the projection onto the space orthogonal to $\widehat{\mathbf{C}}_{1}$. We have

$$
\begin{aligned}
\operatorname{Tr}\left(\mathbf{Q}^{*}\right) & =\operatorname{Tr}\left(\widehat{\mathbf{S}}^{\frac{1}{2}} \mathbf{P}_{\widehat{\mathbf{C}}_{1}} \widehat{\mathbf{S}}^{\frac{1}{2}}\right) \\
& =\operatorname{Tr}\left(\widehat{\mathbf{S}} \mathbf{P}_{\widehat{\mathbf{C}}_{1}}\right) \\
& =\operatorname{Tr}\left(\widehat{\mathbf{S}} \mathbf{P}_{\widehat{\mathbf{C}}_{1}} \mathbf{P}_{\widehat{\mathbf{C}}_{1}}\right) \\
& =\operatorname{Tr}\left(\mathbf{P}_{\widehat{\mathbf{C}}_{1}} \widehat{\mathbf{S}} \mathbf{P}_{\widehat{\mathbf{C}}_{1}}\right)
\end{aligned}
$$

where (15) comes from the fact that for any $\mathbf{A} \in \mathbb{C}^{a \times b}$ and $\mathbf{B} \in \mathbb{C}^{b \times a}, \operatorname{Tr}(\mathbf{A B})=\operatorname{Tr}(\mathbf{B A})$, and (16) holds because $\mathbf{P}_{\widehat{\mathbf{C}}_{1}}=\mathbf{P}_{\widehat{\mathbf{C}}_{1}} \mathbf{P}_{\widehat{\mathbf{C}}_{1}}$. Similarly we have

$$
\begin{aligned}
\operatorname{Tr}(\widehat{\mathbf{S}}) & =\operatorname{Tr}\left(\left(\mathbf{P}_{\widehat{\mathbf{C}}_{1}}+\mathbf{P}_{\widehat{\mathbf{C}}_{1}}^{\perp}\right) \widehat{\mathbf{S}}\left(\mathbf{P}_{\widehat{\mathbf{C}}_{1}}+\mathbf{P}_{\widehat{\mathbf{C}}_{1}}^{\perp}\right)\right) \\
& =\operatorname{Tr}\left(\mathbf{P}_{\widehat{\mathbf{C}}_{1}} \widehat{\mathbf{S}} \mathbf{P}_{\widehat{\mathbf{C}}_{1}}\right)+\operatorname{Tr}\left(\mathbf{P}_{\widehat{\mathbf{C}}_{1}}^{\perp} \widehat{\mathbf{S}} \mathbf{P}_{\widehat{\mathbf{C}}_{1}}^{\perp}\right) \\
& =\operatorname{Tr}\left(\mathbf{Q}^{*}\right)+\operatorname{Tr}\left(\mathbf{P}_{\widehat{\mathrm{C}}_{1}}^{\perp} \widehat{\mathbf{S}} \mathbf{P}_{\widehat{\mathbf{C}}_{1}}^{\perp}\right)
\end{aligned}
$$

where in (18) we used the facts that $\operatorname{Tr}(\mathbf{A B})=\operatorname{Tr}(\mathbf{B A})$ and $\mathbf{P}_{\widehat{\mathbf{C}}_{1}} \mathbf{P}_{\widehat{\mathbf{C}}_{1}}^{\perp}=\mathbf{0}$, and where (19) results from (17).

Lemma 2 For the optimal $\widehat{\mathbf{S}}$, we have $\operatorname{span}\left\{\widehat{\mathbf{C}}_{1}\right\}=\operatorname{span}\{\widehat{\mathbf{S}}\}$.

Proof: The proof is obtained using (19), and by noting that for the optimal $\widehat{\mathbf{S}}$ we must have $\operatorname{Tr}(\widehat{\mathbf{S}})=\operatorname{Tr}\left(\mathbf{Q}^{*}\right)=P_{t}$. This means that we must have $\operatorname{Tr}\left(\mathbf{P}{\stackrel{\widehat{\mathbf{C}}_{1}}{\perp} \widehat{\mathbf{S}} \mathbf{P}}_{\widehat{\mathbf{C}}_{1}}^{\perp}\right)=0$, or equivalently $\mathbf{P} \underset{\widehat{\mathbf{C}}_{1}}{\perp} \widehat{\mathbf{S}}=\mathbf{0}$, which consequently shows that $\operatorname{span}\{\widehat{\mathbf{S}}\} \subseteq \operatorname{span}\left\{\widehat{\mathbf{C}}_{1}\right\}$. To complete the proof we only need to show that $\operatorname{span}\left\{\widehat{\mathbf{C}}_{1}\right\} \subseteq \operatorname{span}\{\widehat{\mathbf{S}}\}$.

Let $\widehat{\mathbf{c}}_{1}$ denote a column vector of $\widehat{\mathbf{C}}_{1}$, i.e., $\widehat{\mathbf{c}}_{1}$ represents a generalized eigenvector of the pencil $\left(\widehat{\mathbf{S}}^{\frac{1}{2}} \mathbf{H}^{H} \mathbf{H} \widehat{\mathbf{S}}^{\frac{1}{2}}+\right.$ $\left.\mathbf{I}, \widehat{\mathbf{S}}^{\frac{1}{2}} \mathbf{G}^{H} \mathbf{G} \widehat{\mathbf{S}}^{\frac{1}{2}}+\mathbf{I}\right)$ corresponding to a generalized eigenvalue bigger than 1 :

$$
\frac{\widehat{\mathbf{c}}_{1}^{H}\left(\widehat{\mathbf{S}}^{\frac{1}{2}} \mathbf{H}^{H} \mathbf{H} \widehat{\mathbf{S}}^{\frac{1}{2}}+\mathbf{I}\right) \widehat{\mathbf{c}}_{1}}{\widehat{\mathbf{c}}_{1}^{H}\left(\widehat{\mathbf{S}}^{\frac{1}{2}} \mathbf{G}^{H} \mathbf{G} \widehat{\mathbf{S}}^{\frac{1}{2}}+\mathbf{I}\right) \widehat{\mathbf{c}}_{1}}>1 .
$$

We can write $\widehat{\mathbf{c}}_{1}$ as $\widehat{\mathbf{c}}_{1}=\mathbf{P}_{\widehat{\mathbf{S}}} \widehat{\mathbf{c}}_{1}+\mathbf{P} \frac{\perp}{\widehat{\mathbf{S}}} \widehat{\mathbf{c}}_{1}$, where $\mathbf{P}_{\widehat{\mathbf{S}}}=$ $\widehat{\mathbf{S}}\left(\widehat{\mathbf{S}}^{H} \widehat{\mathbf{S}}\right)^{-1} \widehat{\mathbf{S}}^{H}$ is the projection matrix onto the space of 
$\widehat{\mathbf{S}}$, and $\mathbf{P}_{\widehat{\mathrm{S}}}^{\perp}=\mathbf{I}-\mathbf{P}_{\widehat{\mathrm{S}}}$ is the projection onto the space orthogonal to $\widehat{\mathbf{S}}$. Letting $a=\widehat{\mathbf{c}}_{1}^{H} \widehat{\mathbf{S}}^{\frac{1}{2}} \mathbf{H}^{H} \mathbf{H} \widehat{\mathbf{S}}^{\frac{1}{2}} \widehat{\mathbf{c}}_{1}$ and $b=$ $\widehat{\mathbf{c}}_{1}^{H} \widehat{\mathbf{S}}^{\frac{1}{2}} \mathbf{G}^{H} \mathbf{G} \widehat{\mathbf{S}}^{\frac{1}{2}} \widehat{\mathbf{c}}_{1}$, we note that $a>b$, and the only component of $\widehat{\mathbf{c}}_{1}$ that affects the values of $a$ and $b$ is $\mathbf{P}_{\widehat{\mathrm{S}}} \widehat{\mathbf{c}}_{1}$. Now, one can easily show that when $a>b$, for any $x$ and $y \geq 0$, we have

$$
\frac{a+x}{b+x} \geq \frac{a+x+y}{b+x+y}
$$

where equality holds if $y=0$. Noting that $\widehat{\mathbf{c}}_{1}^{H} \widehat{\mathbf{c}}_{1}=$ $\widehat{\mathbf{c}}_{1}^{H} \mathbf{P}_{\widehat{\mathbf{S}}} \widehat{\mathbf{c}}_{1}+\widehat{\mathbf{c}}_{1}^{H} \mathbf{P} \widehat{\widehat{\mathbf{S}}^{\prime}} \widehat{\mathbf{c}}_{1}=x+y$, the above point implies that the generalized eigenvalue corresponding to $\widehat{\mathbf{c}}_{1}$ is maximized when $\mathbf{P} \frac{\perp}{\widehat{\mathrm{S}}} \widehat{\mathbf{c}}_{1}=\mathbf{0}$. Consequently, for the optimal $\widehat{\mathbf{S}}$ we must have $\mathbf{P} \underset{\widehat{\mathbf{S}}}{\perp} \widehat{\mathbf{C}}_{1}=\mathbf{0}$, i.e., $\operatorname{span}\left\{\widehat{\mathbf{C}}_{1}\right\} \subseteq \operatorname{span}\{\widehat{\mathbf{S}}\}$, which completes the proof.

Using Lemma 2 in (14), and noting that $\operatorname{span}\left\{\widehat{\mathbf{S}}^{\frac{1}{2}}\right\}=$ $\operatorname{span}\{\widehat{\mathbf{S}}\}=\operatorname{span}\left\{\widehat{\mathbf{C}}_{1}\right\}$, we have

$$
\mathrm{Q}^{*}=\widehat{\mathrm{S}} \text {. }
$$

The following lemma reveals another property of the optimal input covariance matrix under the average power constraint.

Lemma 3 For the optimal $\widehat{\mathbf{S}}$, i.e. $\mathbf{Q}^{*}$, the pencil $\left(\widehat{\mathbf{S}}^{\frac{1}{2}} \mathbf{H}^{H} \mathbf{H} \widehat{\mathbf{S}}^{\frac{1}{2}}+\right.$ $\left.\mathbf{I}, \widehat{\mathbf{S}}^{\frac{1}{2}} \mathbf{G}^{H} \mathbf{G} \widehat{\mathbf{S}}^{\frac{1}{2}}+\mathbf{I}\right)$ has no generalized eigenvalue less than one.

Proof: Let $\widehat{b}$ denote the number of generalized eigenvalues of the pencil $\left(\widehat{\mathbf{S}}^{\frac{1}{2}} \mathbf{H}^{H} \mathbf{H} \widehat{\mathbf{S}}^{\frac{1}{2}}+\mathbf{I}, \widehat{\mathbf{S}}^{\frac{1}{2}} \mathbf{G}^{H} \mathbf{G} \widehat{\mathbf{S}}^{\frac{1}{2}}+\mathbf{I}\right)$ that are strictly bigger than 1 . We show that the rest of the generalized eigenvalues $\left(n_{t}-\widehat{b}\right)$ are equal to one. From Lemma 2, we have $\operatorname{rank}(\widehat{\mathbf{S}})=\operatorname{rank}\left(\widehat{\mathbf{C}}_{1}\right)=\widehat{b}$, where $\widehat{\mathbf{C}}_{1}$ is a $n_{t} \times \widehat{b}$ matrix representing generalized eigenvectors corresponding to generalized eigenvalues bigger than 1 . Also define the $n_{t} \times n_{t}-\widehat{b}$ matrix $\widehat{\mathbf{C}}_{2}=\left[\begin{array}{lll}\widehat{\mathbf{c}}_{21} & \ldots & \widehat{\mathbf{c}}_{2 n_{t}-\widehat{b}}\end{array}\right]$, where $\left\{\widehat{\mathbf{c}}_{2 i}\right\}_{i=1}^{n_{t}-\widehat{b}}$ represent an orthonormal basis for $\operatorname{span}\{\widehat{\mathbf{S}}\}^{\perp}$. From the definition of the generalized eigenvalue decomposition, we note that $\widehat{\mathbf{c}}_{2 i}$, $i=1, \cdots, n_{t}-\widehat{b}$, is a generalized eigenvector corresponding to a generalized eigenvalue equal to 1 . Mathematically, we have

$$
\left(\widehat{\mathbf{S}}^{\frac{1}{2}} \mathbf{H}^{H} \mathbf{H} \widehat{\mathbf{S}}^{\frac{1}{2}}+\mathbf{I}\right) \widehat{\mathbf{c}}_{2 i}=1 \times\left(\widehat{\mathbf{S}}^{\frac{1}{2}} \mathbf{G}^{H} \mathbf{G} \widehat{\mathbf{S}}^{\frac{1}{2}}+\mathbf{I}\right) \widehat{\mathbf{c}}_{2 i} .
$$

Thus for the optimal $\widehat{\mathbf{S}}$, all generalized eigenvectors of the pencil $\left(\widehat{\mathbf{S}}^{\frac{1}{2}} \mathbf{H}^{H} \mathbf{H} \widehat{\mathbf{S}}^{\frac{1}{2}}+\mathbf{I}, \widehat{\mathbf{S}}^{\frac{1}{2}} \mathbf{G}^{H} \mathbf{G} \widehat{\mathbf{S}}^{\frac{1}{2}}+\mathbf{I}\right)$ correspond to generalized eigenvalues either bigger than or equal to 1 . We have:

$$
\begin{aligned}
& \widehat{\mathbf{C}}^{H}\left[\widehat{\mathbf{S}}^{\frac{1}{2}} \mathbf{H}^{H} \mathbf{H} \widehat{\mathbf{S}}^{\frac{1}{2}}+\mathbf{I}\right] \widehat{\mathbf{C}}=\left[\begin{array}{cc}
\widehat{\boldsymbol{\Lambda}}_{1} & \mathbf{0} \\
\mathbf{0} & \mathbf{I}
\end{array}\right] \\
& \widehat{\mathbf{C}}^{H}\left[\widehat{\mathbf{S}}^{\frac{1}{2}} \mathbf{G}^{H} \mathbf{G} \widehat{\mathbf{S}}^{\frac{1}{2}}+\mathbf{I}\right] \widehat{\mathbf{C}}=\left[\begin{array}{ll}
\mathbf{I} & \mathbf{0} \\
\mathbf{0} & \mathbf{I}
\end{array}\right]
\end{aligned}
$$

where $\widehat{\mathbf{C}}=\left[\begin{array}{ll}\widehat{\mathbf{C}}_{1} & \widehat{\mathbf{C}}_{2}\end{array}\right]$ is the generalized eigenvector matrix, and $\widehat{\Lambda}_{1}$ is a $\widehat{b} \times \widehat{b}$ diagonal matrix with diagonal elements representing generalized eigenvalues greater that 1 .

\section{2. rank property}

In the following, another property of the optimal $\mathbf{Q}^{*}$ is revealed. From (21), a straightforward computation yields

$$
\widehat{\mathbf{C}}^{H} \widehat{\mathbf{S}}^{\frac{1}{2}}\left[\mathbf{H}^{H} \mathbf{H}-\mathbf{G}^{H} \mathbf{G}\right] \widehat{\mathbf{S}}^{\frac{1}{2}} \widehat{\mathbf{C}}=\left[\begin{array}{cc}
\widehat{\mathbf{\Lambda}}_{1}-\mathbf{I} & \mathbf{0} \\
\mathbf{0} & \mathbf{0}
\end{array}\right] \succeq \mathbf{0},
$$

from which we have

$$
\widehat{\mathbf{C}}_{1}^{H} \widehat{\mathbf{S}}^{\frac{1}{2}}\left[\mathbf{H}^{H} \mathbf{H}-\mathbf{G}^{H} \mathbf{G}\right] \widehat{\mathbf{S}}^{\frac{1}{2}} \widehat{\mathbf{C}}_{1}=\widehat{\mathbf{\Lambda}}_{1}-\mathbf{I} \succ \mathbf{0} .
$$

Let $\widehat{b}$ denote $\widehat{b}=\operatorname{rank}\left(\mathbf{Q}^{*}\right)$. We note from lemma 2 that $\operatorname{rank}\left(\mathbf{Q}^{*}\right)=\operatorname{rank}\left(\widehat{\mathbf{C}}_{1}\right)=\operatorname{rank}\left(\widehat{\mathbf{\Lambda}}_{1}\right)=\widehat{b}$. Denote the singular value decomposition of $\widehat{\mathbf{C}}_{1}$ as

$$
\widehat{\mathbf{C}}_{1}=\mathbf{U}_{c} \mathbf{D}_{c} \mathbf{V}_{c}^{H},
$$

where $\mathbf{U}_{c}$ is a $n_{t} \times \widehat{b}$ matrix whose columns are an orthonormal basis for $\operatorname{span}\left\{\widehat{\mathbf{C}}_{1}\right\}, \mathbf{U}_{c}^{H} \mathbf{U}_{c}=\mathbf{I}, \mathbf{D}_{c}$ is a $\widehat{b} \times \widehat{b}$ positive definite diagonal matrix, and $\mathbf{V}_{c}$ is a unitary matrix. Recall from lemma 2 that $\operatorname{span}\left\{\widehat{\mathbf{C}}_{1}\right\}=\operatorname{span}\left\{\mathbf{Q}^{*}\right\}$, the eigenvalue decomposition of $\mathbf{Q}^{*}$ is written as

$$
\widehat{\mathbf{S}}=\mathbf{Q}^{*}=\mathbf{U}_{c} \mathbf{D}_{q} \mathbf{U}_{c}^{H},
$$

where $\mathbf{D}_{q} \succ \mathbf{0}$ is a $\widehat{b} \times \widehat{b}$ diagonal matrix. Using (23) and (24) in (22), we have

$$
\begin{aligned}
\mathbf{V}_{c} \mathbf{D}_{c} \mathbf{D}_{q}^{\frac{1}{2}} \mathbf{U}_{c}^{H}\left[\mathbf{H}^{H} \mathbf{H}-\mathbf{G}^{H} \mathbf{G}\right] \mathbf{U}_{c} \mathbf{D}_{q}^{\frac{1}{2}} \mathbf{D}_{c} \mathbf{V}_{c}^{H} & \\
& =\widehat{\boldsymbol{\Lambda}}_{1}-\mathbf{I} \succ \mathbf{0},
\end{aligned}
$$

which results in

$$
\begin{aligned}
\mathbf{U}_{c}^{H} & {\left[\mathbf{H}^{H} \mathbf{H}-\mathbf{G}^{H} \mathbf{G}\right] \mathbf{U}_{c} } \\
& =\mathbf{D}_{q}^{-\frac{1}{2}} \mathbf{D}_{c}^{-1} \mathbf{V}_{c}^{H}\left(\widehat{\boldsymbol{\Lambda}}_{1}-\mathbf{I}\right) \mathbf{V}_{c} \mathbf{D}_{c}^{-1} \mathbf{D}_{q}^{-\frac{1}{2}} \succ \mathbf{0} .
\end{aligned}
$$

The above equality shows that for the optimal $\mathbf{Q}^{*}$ with $\widehat{b}=$ $\operatorname{rank}\left(\mathbf{Q}^{*}\right), \mathbf{U}_{c}^{H}\left[\mathbf{H}^{H} \mathbf{H}-\mathbf{G}^{H} \mathbf{G}\right] \mathbf{U}_{c}$ is a $\widehat{b} \times \widehat{b}$ positive definite matrix.

Theorem 1 For the optimal $\mathbf{Q}^{*}$, i.e. $\widehat{\mathbf{S}}$, we have

$$
\operatorname{rank}\left(\mathbf{Q}^{*}\right) \leq m
$$

where $m$ is the number of positive eigenvalues of the matrix $\mathbf{H}^{H} \mathbf{H}-\mathbf{G}^{H} \mathbf{G}$.

Proof: Letting $\widehat{b}=\operatorname{rank}\left(\mathbf{Q}^{*}\right)$, we want to show that $\widehat{b} \leq$ $m$. To prove that $\operatorname{rank}\left(\mathbf{Q}^{*}\right)=\widehat{b} \leq m$, we only need to show that there is no $\mathbf{U}_{c} \in \mathbb{C}^{n_{t} \times \widehat{b}}, \mathbf{U}_{c}^{H} \mathbf{U}_{c}=\mathbf{I}$, such that $\mathbf{U}_{c}^{H}\left[\mathbf{H}^{H} \mathbf{H}-\mathbf{G}^{H} \mathbf{G}\right] \mathbf{U}_{c} \succ \mathbf{0}$ and $\widehat{b}>m$. The proof is obtained by contradiction. We assume $\widehat{b}>m$ and show that under this assumption, there exist specific non-zero vectors $\mathbf{e}_{x}$ 
for which $\mathbf{e}_{x}^{H} \mathbf{U}_{c}^{H}\left[\mathbf{H}^{H} \mathbf{H}-\mathbf{G}^{H} \mathbf{G}\right] \mathbf{U}_{c} \mathbf{e}_{x}=0$, which contradicts the fact that (25) is positive definite.

Denote the eigenvalue decomposition of $\mathbf{H}^{H} \mathbf{H}-\mathbf{G}^{H} \mathbf{G}$ as

$$
\mathbf{H}^{H} \mathbf{H}-\mathbf{G}^{H} \mathbf{G}=\sum_{i=1}^{m} \lambda_{i}^{+} \mathbf{f}_{i} \mathbf{f}_{i}^{H}+\sum_{i=m+1}^{n_{t}} \lambda_{i}^{-} \mathbf{f}_{i} \mathbf{f}_{i}^{H},
$$

where $\lambda_{i}^{+}, \lambda_{i}^{-}$respectively represent positive and non-positive eigenvalues, we have

$$
\begin{aligned}
\mathbf{U}_{c}^{H}\left[\mathbf{H}^{H} \mathbf{H}-\mathbf{G}^{H} \mathbf{G}\right] \mathbf{U}_{c} & =\sum_{i=1}^{m} \lambda_{i}^{+} \mathbf{t}_{i} \mathbf{t}_{i}^{H} \\
& +\sum_{i=m+1}^{n_{t}} \lambda_{i}^{-} \mathbf{t}_{i} \mathbf{t}_{i}^{H} \succ \mathbf{0},
\end{aligned}
$$

where $\mathbf{t}_{i}=\mathbf{U}_{c}^{H} \mathbf{f}_{i} \in \mathbb{C}^{\widehat{b} \times 1}$. Assuming $\widehat{b}>m$, define $\mathbf{T}^{+}=$ $\left[\mathbf{t}_{1} \ldots \mathbf{t}_{m}\right]$. Let $\mathbf{P}_{\mathbf{T}^{+}}$denote the projection matrix on to the space of $\mathbf{T}^{+}$, and let $\widehat{r}=\operatorname{rank}\left(\mathbf{T}^{+}\right)$. We note that any $\mathbf{t}_{i}$ corresponding to $\lambda_{i}^{-}$can be written as

$$
\mathbf{t}_{i}=\mathbf{P}_{\mathbf{T}^{+}} \mathbf{t}_{i}+\sum_{j=1}^{\widehat{b}-\widehat{r}} v_{i j} \mathbf{e}_{j} \quad i=m+1, \ldots n_{t},
$$

where $\left\{\mathbf{e}_{j}\right\}_{j=1}^{j=\widehat{b}-\widehat{r}}$ is a set of orthonormal basis vectors for $\operatorname{span}\left\{\mathbf{T}^{+}\right\}^{\perp}$, and $v_{i j}$ is a complex scalar. Noting that $\mathbf{e}_{k}^{H} \mathbf{e}_{j}=1$ if $j=k$ and $\mathbf{e}_{k}^{H} \mathbf{e}_{j}=0$ when $j \neq k$, one can easily confirm that

$$
\begin{gathered}
\mathbf{e}_{k}^{H} \mathbf{U}_{c}^{H}\left[\mathbf{H}^{H} \mathbf{H}-\mathbf{G}^{H} \mathbf{G}\right] \mathbf{U}_{c} \mathbf{e}_{k}=\sum_{i=m+1}^{i=n_{t}} \lambda_{i}^{-}\left|v_{i k}\right|^{2}>0 \\
k=1, \ldots, \widehat{b}-\widehat{r}
\end{gathered}
$$

Recalling that $\lambda_{i}^{-} \leq 0$, (27) leads to a contradiction since the right side of (27) is zero (after setting $v_{i k}=0$ for nonzero $\lambda_{i}^{-}$), while the left side must be positive. This contradiction comes from the guaranteed existence of non-zero vectors $\left\{\mathbf{e}_{j}\right\}_{j=1}^{j=\widehat{b}-\widehat{r}}$ when $\widehat{b}$ is assumed to be bigger than $m$. Thus, $\operatorname{rank}\left(\mathbf{Q}^{*}\right)=\widehat{b} \leq m$.

Remark 2 From Theorem 1, one can easily confirm that the optimal $\mathbf{Q}^{*}$ can be full rank only in the case that $m=n_{t}$, i.e. $\mathbf{H}^{H} \mathbf{H} \succ \mathbf{G}^{H} \mathbf{G}$. For all other scenarios, the optimal $\mathbf{Q}^{*}$ will be low rank.

\section{REFERENCES}

[1] A. Wyner, "The wire-tap channel," Bell. Syst. Tech. J., vol. 54, no. 8, pp. 1355-1387, Jan. 1975.

[2] S. K. Leung-Yan-Cheong and M. E. Hellman, "The Gaussian wire-tap channel," IEEE Trans. Inf. Theory, vol. 24, pp. 451-456, Jul. 1978.
[3] F. Oggier and B. Hassibi, "The secrecy capacity of the MIMO wiretap channel," in Proc. IEEE Int. Symp. Information Theory Toronto, ON, Canada, Jul. 2008, pp. 524-528.

[4] A. Khisti and G. Wornell, "Secure transmission with multiple antennas II: The MIMOME wiretap channel," IEEE Trans. Inf. Theory, vol. 56, no. 11, pp. 5515-5532, 2010.

[5] J. Li and A. P. Petropulu, "Transmitter optimization for achieving secrecy capacity in Gaussian MIMO wiretap channels," submitted to IEEE Trans. Info. Theory, Available [online]: http://arxiv.org/PS cache/arxiv/pdf/0909/0909.2622v1.pdf.

[6] A. Khisti and G. Wornell, "Secure transmission with multiple antennas I: The MISOME wiretap channel," IEEE Trans. Inf. Theory, vol. 56, no. 7, pp. 3088-3104, 2010.

[7] S. Shafiee and S. Ulukus, "Towards the Secrecy Capacity of the Gaussian MIMO Wire-Tap Channel: The 2-2-1 Channel," IEEE Trans. on Inf. Theory, vol. 55, no. 9, Sep. 2009.

[8] T. Liu and S. Shamai (Shitz), "A note on secrecy capacity of the multi-antenna wiretap channel," IEEE Trans. Inf. Theory, vol. 55, no. 6, pp. 2547-2553, 2009.

[9] R. Bustin, R. Liu, H. V. Poor, and S. Shamai (Shitz), "A MMSE approach to the secrecy capacity of the MIMO Gaussian wiretap channel," EURASIP Journal on Wireless Communications and Networking, vol. 2009, Article ID 370970, 8 pages, 2009.

[10] H. Weingarten, Y. Steinberg, and S. Shamai (Shitz), "The capacity region of the Gaussian multiple-input multipleoutput broadcast channel," IEEE Trans. Inf. Theory, vol. 52, no. 9, pp. 3936-3964, 2006.

[11] R. Liu, T. Liu, H. V. Poor, and S. Shamai, "Multipleinput multiple-output Gaussian broadcast channels with confidential messages," IEEE Trans. Inf. Theory, vol. 56, no. 9, pp. 4215-4227, 2010.

[12] R. A. Horn and C. R. Johnson, Matrix Analysis, University Press, Cambridge, UK, 1999. 